\title{
Upregulation of heme oxygenase-1 protects genetically fat Zucker rat livers from ischemia/reperfusion injury
}

\author{
Farin Amersi, ${ }^{1}$ Roland Buelow, ${ }^{2}$ Hirohisa Kato, ${ }^{1}$ Bibo Ke, ${ }^{1}$ Ana J. Coito, ${ }^{1}$ Xiu-Da Shen, ${ }^{1}$ \\ Delai Zhao, ${ }^{1}$ Joseph Zaky, ${ }^{1}$ Judy Melinek, ${ }^{3}$ Charles R. Lassman, ${ }^{3}$ Jay K. Kolls, ${ }^{4}$ \\ J. Alam, ${ }^{5}$ Thomas Ritter, ${ }^{6}$ Hans-Dieter Volk, ${ }^{6}$ Douglas G. Farmer, ${ }^{1}$ \\ Rafik M. Ghobrial, ${ }^{1}$ Ronald W. Busuttil, ${ }^{1}$ and Jerzy W. Kupiec-Weglinski ${ }^{1}$
}

\footnotetext{
${ }^{1}$ The Dumont-UCLA Transplant Center, Division of Liver and Pancreas Transplantation, Department of Surgery, and ${ }^{2}$ Sangstat Medical Corporation, Fremont, California 94555, USA

${ }^{3}$ Department of Pathology and Laboratory Medicine, UCLA School of Medicine, Los Angeles, California 90095, USA

${ }^{4}$ Section of Pulmonary/Critical Care, Louisiana State University School of Medicine, New Orleans, Louisiana 70112, USA

${ }^{5}$ Department of Molecular Genetics, Alton Ochsner Medical Foundation, and Department of Biochemistry/Molecular Biology, Louisiana State University Medical Center, New Orleans, Louisiana 70121, USA

${ }^{6}$ Institute of Medical Immunology, Humboldt University, D-10098 Berlin, Germany
}

Address correspondence to: Jerzy W. Kupiec-Weglinski, The Dumont-UCLA Transplant Center, Room 77-120 CHS, 10833 Le Conte Avenue, Los Angeles, California 90095, USA.

Phone: (310) 825-4196; Fax: (310) 267-2358; E-mail: jkupiecw@surgery.medsch.ucla.edu.

Received for publication July 19, 1999, and accepted in revised form October 18, 1999.

\begin{abstract}
We examined the effects of upregulation of heme oxygenase-1 (HO-1) in steatotic rat liver models of ex vivo cold ischemia/reperfusion (I/R) injury. In the model of ischemia/isolated perfusion, treatment of genetically obese Zucker rats with the HO-1 inducer cobalt protoporphyrin (CoPP) or with adenoviral HO-1 (Ad-HO-1) significantly improved portal venous blood flow, increased bile production, and decreased hepatocyte injury. Unlike in untreated rats or those pretreated with the HO1 inhibitor zinc protoporphyrin ( $\mathrm{ZnPP}$ ), upregulation of HO-1 by Western blots correlated with amelioration of histologic features of $\mathrm{I} / \mathrm{R}$ injury. Adjunctive infusion of $\mathrm{ZnPP}$ abrogated the beneficial effects of Ad-HO-1 gene transfer, documenting the direct involvement of HO-1 in protection against $\mathrm{I} / \mathrm{R}$ injury. Following cold ischemia/isotransplantation, $\mathrm{HO}-1$ overexpression extended animal survival from $40 \%$ in untreated controls to about $80 \%$ after CoPP or Ad-HO- 1 therapy. This effect correlated with preserved hepatic architecture, improved liver function, and depressed infiltration by $T$ cells and macrophages. Hence, CoPP- or gene therapy-induced HO-1 prevented I/R injury in steatotic rat livers. These findings provide the rationale for refined new treatments that should increase the supply of usable donor livers and ultimately improve the overall success of liver transplantation.
\end{abstract}

J. Clin. Invest. 104:1631-1639 (1999).

\section{Introduction}

Orthotopic liver transplantation (OLT) is an effective therapeutic modality for end-stage liver disease. Advances in surgical technique, patient management, and immunosuppression have improved patient survival after OLT, resulting in expansion of the list of indications for the procedure. However, a serious problem limiting OLT is the widening disparity between the increasing number of potential recipients who vie for a constant donor supply. This demand has renewed interest in the use of marginal livers to expand the number of available donors. The prevalence of fatty infiltration in the liver ranges from 6 to $26 \%$ based on autopsy studies and donor liver biopsies $(1,2)$. Indeed, steatotic donor livers are frequently discarded because of the fear of primary nonfunction, as compared with normal livers, further accentuating the critical shortage of human donor livers $(3,4)$. Because $10-30 \%$ of potential liver recipients on transplant waiting lists die without being transplanted, considerable effort has been made to identify methods that would allow successful use of steatotic grafts.

Ischemia/reperfusion (I/R) injury, an antigen-independent event, often leads to primary nonfunction or early dysfunction of steatotic liver grafts. Moreover, ischemia of more than 12 hours strongly correlates not only with primary nonfunction after OLT, but also with biopsy-proven centrilobular necrosis and a higher incidence of both acute and chronic rejection (5). The mechanism of liver injury following I/R involves a complex interaction of events, which include neutrophil activation, increased expression of adhesion molecules, Kupffer cell activation, cytokine release, sinusoidal endothelial cell death, and hepatocyte injury $(6,7)$. Hepatocytes are more susceptible to ischemic injury with damage to proteins, lipids, and DNA, altered regulation of signal transduction, and release of growth factors (8). The exact mechanism that leads to the ulti- 
mate decline in liver function and eventual organ failure remains elusive, but data suggest that the oxidative stress of I/R plays a major role (9). The consequences of oxidative stress include lipid peroxidation, as well as damage to proteins and nucleic acids. Because of the nature of liver disease, transplant patients may be further disadvantaged by starting from a baseline deletion of normal antioxidative mechanisms. Normal cellular antioxidant systems involve a delicate balance of several enzymes and proteins that are increasingly recognized as critical regulators of cell function and viability (10).

Heme oxygenases $(\mathrm{HO})$ are the rate-limiting enzymes that catalyze the conversion of heme into biliverdin, carbon monoxide (CO), and free iron (11). They consist of 3 isoforms, the inducible HO-1, also known as heat shock protein 32 (hsp32), the constitutive $\mathrm{HO}-2$, and the not fully defined HO-3. HO-1 activity is upregulated following various stimuli and is considered one of the most sensitive indicators of cellular stress. Interestingly, HO-1 levels may also increase in animals after administration of $\mathrm{HO}$ inhibitors, perhaps because of the accumulation of intracellular heme upon inhibition of $\mathrm{HO}$ (12). In analogy with heat-shock regulation, upregulation of HO-1 may be an adaptive mechanism protecting cells from stress, in particular hyperoxia, ischemia, or inflammation.

In transplantation models increased expression of HO-1 was associated with long-term acceptance of cardiac xenografts, whereas donor hearts from HO-1-deficient donors were vigorously rejected (13). In our own studies, pretreatment of mice with cobalt protoporphyrin (CoPP), a known HO-1 inducer, prolonged cardiac allograft survival (14), whereas exogenous HO-1 overexpression prevented the development of cardiac transplant arteriosclerosis and interstitial fibrosis characteristic of chronic rejection (15). Moreover, a recent study demonstrated that induction of HO-1 was neuroprotective and attenuated cellular injury caused by ischemic stroke (16). Hence, pharmacological stimulation of HO-1 activity may represent a novel therapeutic approach in the amelioration of ischemic injury during the acute period of stroke.

This study was designed to determine the role of HO1 expression in well-defined steatotic rat liver models of ex vivo cold ischemia followed by perfusion or syngeneic OLT. To our knowledge, we have demonstrated for the first time that overexpression of HO-1 after pretreatment with CoPP or gene therapy with a recombinant adenovirus encoding rat HO-1 cDNA (Ad-HO-1) can prevent severe I/R insult suffered by steatotic livers. The beneficial effects upon hepatocyte injury and liver function were abrogated after adjunctive infusion of zinc protoporphyrin ( $\mathrm{ZnPP})$, the HO-1 antagonist, which implicates a direct involvement of $\mathrm{HO}-1$ in the protection against $\mathrm{I} / \mathrm{R}$ injury. Our present findings raise the possibility of refined new treatment regimens, which, in turn, may increase the OLT donor pool through modulation of marginal livers and ultimately improve the overall success of liver transplantation.

\section{Methods}

Animals. Genetically obese $(f a / f a)$ male Zucker rats (220-275 g) and lean ( $f a /-)$ Zucker rats (250-300 g) were used (Harlan Sprague Dawley Inc., Indianapolis, Indiana, USA). Animals were fed standard rodent chow and water ad libitum and cared for according to guidelines approved by the American Association of Laboratory Animal Care.

Synthetic metalloporphyrins. Metalloporphyrins (CoPP and $\mathrm{ZnPP}$ ) were purchased from Porphyrin Products Inc. (Logan, Utah, USA). They were dissolved in $0.2 \mathrm{M}$ $\mathrm{NaOH}$, subsequently adjusted to a $\mathrm{pH}$ of 7.4 , and diluted in $0.85 \% \mathrm{NaCl}$. The stock concentration of metalloporphyrins was $1 \mathrm{mg} / \mathrm{mL}$.

Ad-HO-1 construct. A 1.0-kbp XhoI-HindIII fragment from the rat HO-1 cDNA clone pRHO-1 (17), containing the entire coding region was cloned into plasmid pAC-CMVpLpA $(18,19)$. Ad-HO-1 was generated by homologous recombination in 911 cells (20) after cotransfection with the $\mathrm{PAC}-\mathrm{HO}-1$ plasmid and plasmid pJM17 (21). The recombinant Ad-HO-1 clones were screened by Southern blot analysis. Ad containing the Escherichia coli $\beta$-galactosidase gene (Ad- $\beta \mathrm{Gal}$ ) has been described (22).

Ex vivo cold ischemia model. Genetically obese Zucker rats underwent ether anesthesia and systemic heparinization. After skeletonization of the liver, the portal vein, bile duct, and inferior vena cava were cannulated, and the liver was flushed with $10 \mathrm{~mL}$ of University of Wisconsin (UW) solution. Control livers from untreated obese Zucker rats were stored for 6 hours at $4^{\circ} \mathrm{C}$ in UW solution $(n=6)$. There were 4 treatment groups. Group 1 animals received CoPP, the $\mathrm{HO}-1$ inducer $(5 \mathrm{mg} / \mathrm{kg}$ intraperitoneally) 24 hours before liver harvest $(n=6)$. Group 2 rats were infused with Ad-HO-1 or Ad- $\beta$ Gal $\left(2.4 \times 10^{9}\right.$ plaque-forming units $[\mathrm{pfu}]$ intraperitoneally $)$

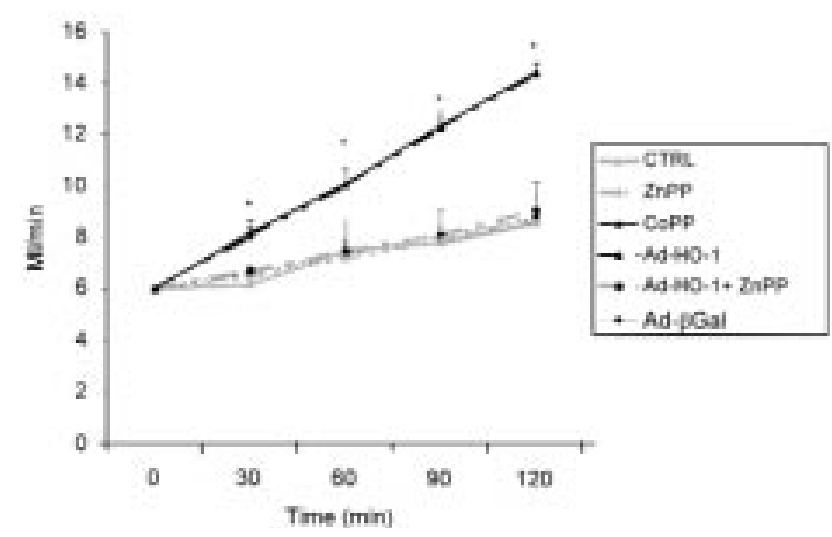

Figure 1

$\mathrm{HO}$-1-inducing agents decrease resistance to portal vein blood flow. Livers harvested from obese Zucker rats were perfused for 2 hours on the isolated perfusion rat liver apparatus after 6 hours of cold ischemia. Pretreatment with CoPP or Ad-HO-1 gene transfer (day-1) significantly improved portal venous blood flow compared with untreated, $\mathrm{ZnPP}$, or Ad- $\beta$ Gal pretreated controls throughout the reperfusion period. These data represent the mean \pm SE of $4-10$ independent perfusions for each group. ${ }^{*} P=0.0001$ versus untreated/ZnPP-treated controls. 


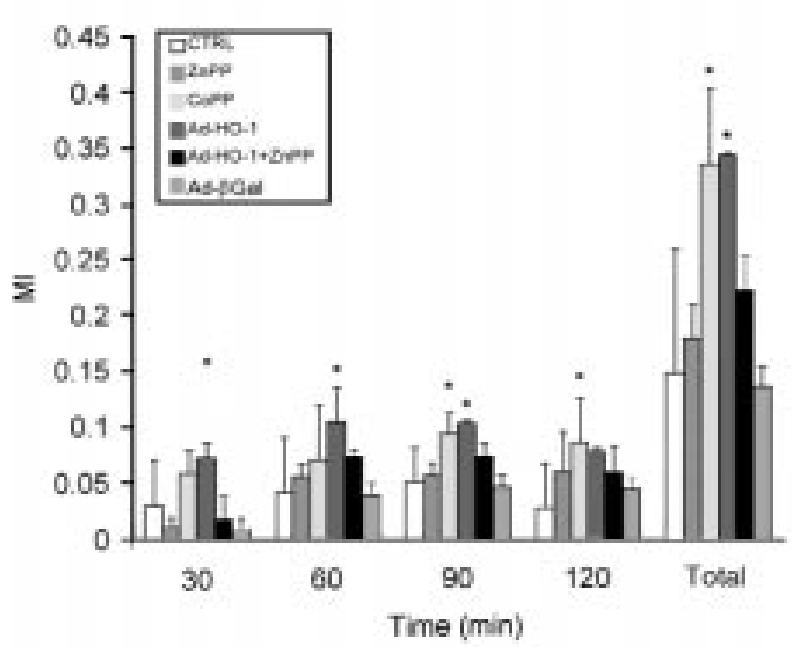

Figure 2

Bile production in fatty livers perfused for 2 hours on the isolated perfusion rat liver apparatus after 6 hours of cold ischemia. Animals were pretreated with metalloporphyrins, or with Ad-HO-1 gene transfer, or left untreated. Bile production at 30-minute intervals throughout the reperfusion period was significantly higher in the CoPP/Ad-HO-1 groups ( $\left.{ }^{*} P<0.05\right)$ as compared with untreated, $\mathrm{ZnPP}$-, or Ad- $\beta \mathrm{Gal}$-pretreated controls. These data represent the mean \pm SE of 4-10 independent perfusions for each group. ${ }^{*} P<0.05$ versus untreated/ZnPP-treated controls.

24 to 48 hours before the procurement $(n=4-10)$. Group 3 donors were treated with Ad-HO-1 $\left(2.4 \times 10^{9}\right.$ pfu intravenously) at day -2 , followed 1 day later by infusion of $\mathrm{ZnPP}(20 \mathrm{mg} / \mathrm{kg}$ intraperitoneally), the HO-1 inhibitor $(n=4)$. Group 4 rats received $\mathrm{ZnPP}$ alone $(20 \mathrm{mg} / \mathrm{kg}$ intraperitoneally) at 24 hours before harvest $(n=4)$. All livers were procured at day 0 , stored for 6 hours at $4{ }^{\circ} \mathrm{C}$ in UW solution, and then perfused on an isolated perfusion rat liver apparatus, as described (6). The Zucker livers were perfused ex vivo for 2 hours while temperature, $\mathrm{pH}$, and inflow pressure were kept constant. Portal vein blood flow and pressure were recorded every 15 minutes, whereas bile output was monitored every 30 minutes. Portal vein blood flow was adjusted to maintain portal pressures of 13 to $18 \mathrm{cmH}_{2} 0$. Blood was collected at 30minute intervals and serum glutamic-oxaloacetic transaminase (sGOT) levels were measured using an autoanalyzer from ANTECH Diagnostics (Irvine, California, USA). Following 2 hours of perfusion, a portion of the liver was snap-frozen for mRNA extraction and Western blot analysis of HO-1 expression; the remaining tissue samples were fixed in formalin for hematoxylin and eosin (H\&E) staining.

Syngeneic OLT model. Syngeneic liver transplants were performed using fatty livers that were harvested from obese Zucker rats and stored for 4 hours at $4^{\circ} \mathrm{C}$ in UW solution before being transplanted into lean Zucker recipients. OLTs were performed with revascularization without hepatic artery reconstruction (23). There were 2 treatment groups. In the first group, obese Zucker rats ( $n$ $=10)$ received $\operatorname{CoPP}(5 \mathrm{mg} / \mathrm{kg}$ intraperitoneally $) 24$ hours before the procurement. Group 2 donors $(n=11)$ were treated with Ad-HO-1 (2.4 × $10^{9}$ pfu intravenously) 24 hours before harvest. OLT recipients were followed for survival and sGOT levels. Separate groups of rats $(n=$ 2 /group) were sacrificed at $1,7,14$, and 100 days after OLT, and liver samples were collected for H\&E/immunohistology staining and Western blot analysis.

Histology and immunohistochemistry. Liver specimens were fixed in a $10 \%$ buffered formalin solution and embedded in paraffin. Sections were made at $4 \mu \mathrm{m}$ and stained with H\&E. The histologic severity of I/R injury in the ex vivo perfusion model was graded using International Banff Criteria (24). Using these criteria, lobular disarray and ballooning changes are graded from 1 to 4 , where no change is given a score of 1 and severe disarray or ballooning changes is given a score of 4 . The previously published Suzuki's criteria (25) were modified to evaluate the histologic severity of I/R injury in the OLT model. In this classification sinusoidal congestion, hepatocyte necrosis, and ballooning degeneration are graded from 0 to 4 . No necrosis, congestion or centrilobular ballooning is given a score of 0 , whereas severe congestion and ballooning degeneration as well as greater than $60 \%$ lobular necrosis is given a value of 4 .

OLTs were examined serially by immunohistochemistry for mononuclear cell (MNC) infiltration $(26,27)$. Briefly, liver tissue was embedded in Tissue Tek OCT compound (Miles Inc., Elkhart, Indiana, USA), snap frozen in liquid nitrogen, and stored at $-70^{\circ} \mathrm{C}$. Cryostat sections $(5 \mu \mathrm{m})$ were fixed in acetone, and then endogenous peroxidase activity was inhibited with $0.3 \% \mathrm{H}_{2} \mathrm{O}_{2}$ in PBS. Normal heat-inactivated donkey serum (10\%) was used for blocking. Appropriate primary mouse Ab

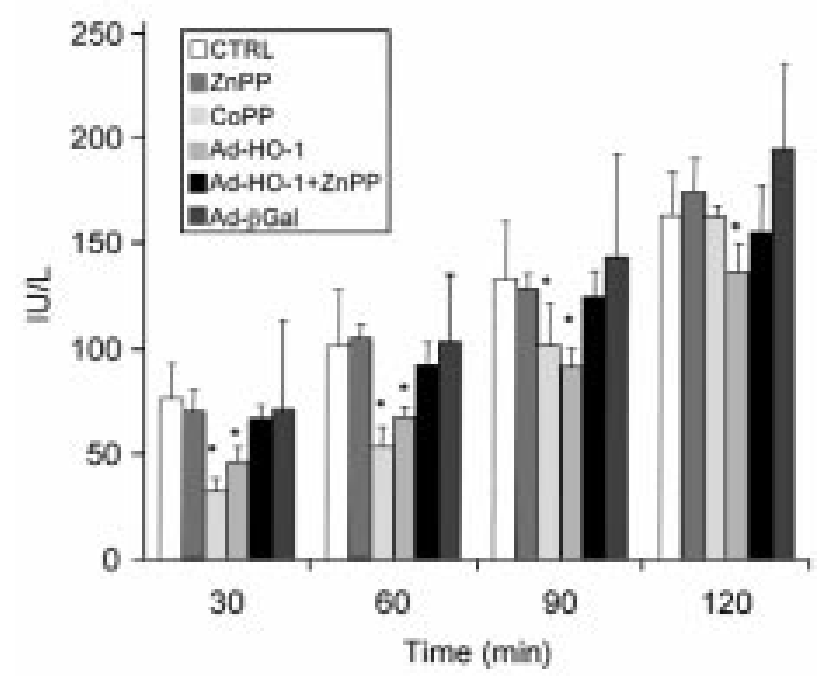

Figure 3

Effects of $\mathrm{I} / \mathrm{R}$ injury on sGOT release from fatty livers cold stored for 6 hours followed by 2 hours of ex vivo perfusion. Levels (IU/L) were measured in blood samples taken at 30-minute intervals during the perfusion. Levels of sGOT were significantly lower at 30,60 , and 90 minutes during the perfusion of livers pretreated with CoPP or Ad-HO-1 as compared with untreated controls or those pretreated with ZnPP or Ad$\beta G$ al. These data represent the mean \pm SE of 4-10 measurements for each group. ${ }^{*} P<0.05$ versus untreated/ZnPP-treated control. 

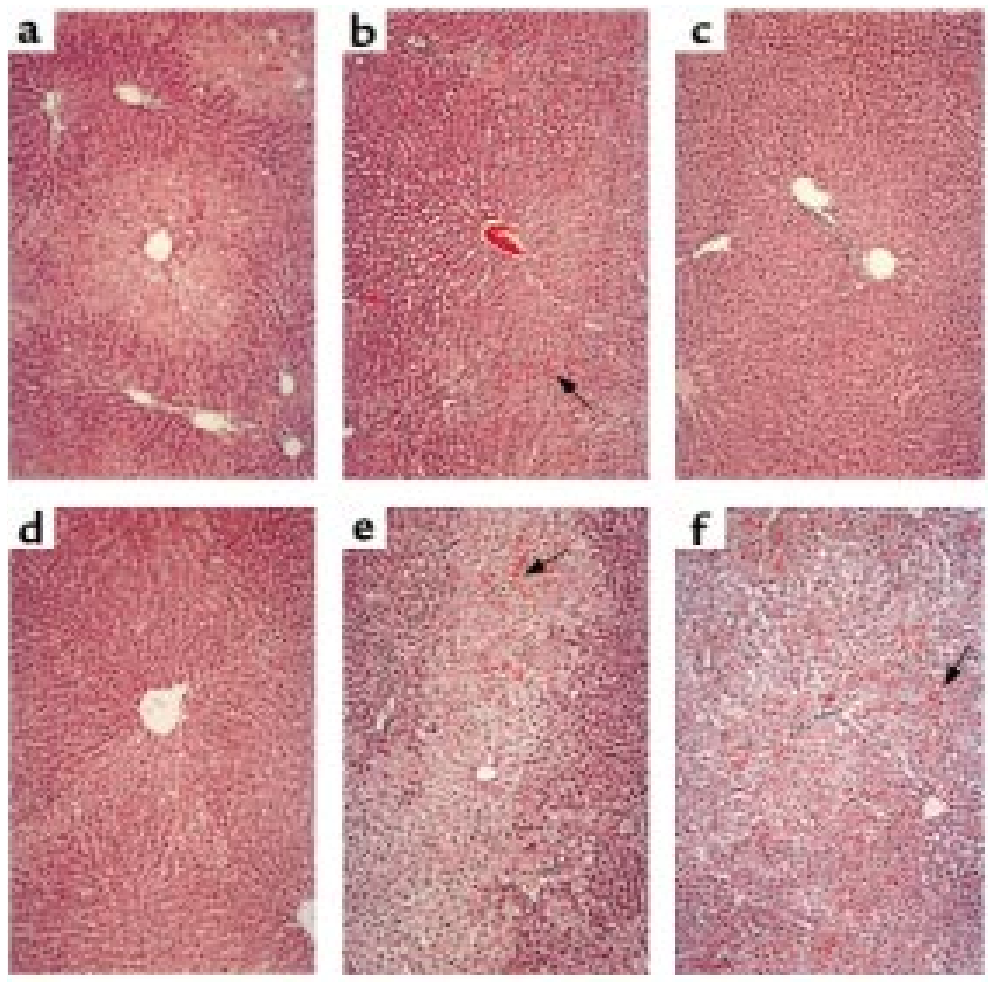

\section{Figure 4}

Photomicrographs of representative rat fatty livers after 6 hours of cold ischemia and 2 hours of reperfusion on the isolated perfusion rat liver apparatus. (a) Control untreated group with severe lobular distortion, zone 3 ballooning, and hepatocyte necrosis (Banff's score $=3.0 \pm 0.63$ ); (b) ZnPP-pretreated group with marked sinusoidal and vascular congestion (arrow) and profound zone 3 ballooning change (score $=2.86 \pm 0.12) ;(\mathbf{c})$ CoPP-pretreated and (d) Ad-HO-1-pretreated groups with minimal vacuolar degeneration and almost complete preservation of lobular architecture (scores $=1.21 \pm 0.39$ and 1.68 \pm 0.51 , respectively); (e) Ad-HO-1 plus ZnPP-treated group, similar to untreated controls, with profound zone 3 ballooning change accompanied by confluent hepatocyte necrosis (arrow; score $=2.74$ \pm 0.26 ); (f) Ad- $\beta$ Gal-pretreated group, similar to untreated controls, showing profound zone 3 ballooning change accompanied by severe vascular congestion and confluent hepatocyte necrosis (arrow; score $=3.0 \pm 1.41) . \times 100, \mathrm{H} \& \mathrm{E}$ stain; $n=2-3$ /group. against rat $\mathrm{T}$ cells (R73) and monocytes/macrophages (ED1) (Harlan Bioproducts for Science, Indianapolis, Indiana, USA) were added at optimal dilutions. Bound primary $\mathrm{Ab}$ was detected using biotinylated donkey anti-mouse IgG and streptavidin peroxidase-conjugated complexes (DAKO Corp., Carpinteria, California, USA). The control sections were performed by replacing the primary $\mathrm{Ab}$ with either dilution buffer or normal mouse serum. The peroxidase reaction was developed with 3,3-diaminobenzidine tetrahydrochloride (Sigma Chemical Co., St. Louis, Missouri, USA). The sections were evaluated blindly by counting the labeled cells in triplicates in 10 high-power fields.

Western blots. Protein was extracted from liver tissue samples with PBSTDS buffer (50 mM Tris, $150 \mathrm{mM}$ $\mathrm{NaCl}, 0.1 \% \mathrm{SDS}, 1 \%$ sodium deoxycholate, and $1 \%$ triton $\mathrm{X}-100, \mathrm{pH} 7.2)$. Proteins (30 $\mu \mathrm{g} / \mathrm{sample})$ in SDSloading buffer (50 mM Tris, pH 7.6, 10\% glycerol, 1\% SDS) were subjected to $12 \%$ SDS-PAGE and transferred to nitrocellulose membrane (Bio-Rad Laboratories Inc., Hercules, California, USA). The gel was then stained with Coomassie blue to document equal protein loading. The membrane was blocked with 3\% dry milk and 0.1\% Tween-20 (Amersham, Arlington Heights, Illinois, USA) in PBS and incubated with rabbit anti-rat HO-1 polyclonal Ab (Sangstat Corp., San Francisco, California, USA). The filters were washed and then incubated with horseradish peroxidase donkey anti-rabbit Ab (Amersham Life Sciences, Arlington Heights, Illinois, USA). Relative quantities of HO1 protein were determined using a densitometer (Kodak Digital Science 1D Analysis Software, Rochester, New York, USA).
Statistics. Results are expressed as mean \pm SEM. Statistical comparisons between the groups in the ex-vivo perfusion model were performed using repeated measure ANOVA. We used the Tukey-Fisher least-significant difference (LSD) criterion for judging statistical significance where $P$ values of less than 0.05 were considered statistically significant.

\section{Results}

The effects of HO-1-inducing agents in the ex vivo steatotic rat liver cold ischemia model followed by reperfusion. To test our hypothesis that overexpression of HO-1 decreases I/Rmediated hepatocyte injury, we monitored portal vein blood flow, bile production, and sGOT levels in livers from obese Zucker rats that were either untreated or pretreated with $\mathrm{HO}-1$-inducing agents and then perfused for 2 hours on the isolated perfusion rat liver apparatus.

Pretreatment of Zucker rats with synthetic metalloporphyrin CoPP or Ad-HO-1 gene transfer exerted equally protective effects against liver I/R injury (Figure 1). Both modalities significantly improved portal blood flow throughout the 2 -hour reperfusion period, as compared with untreated controls $(P=0.0001)$. In addition, as shown in Figure 2, both CoPP and AdHO-1 significantly increased bile production $(P<$ $0.05)$, as compared with controls. The I/R-induced hepatocyte injury measured by sGOT release was also markedly reduced in the CoPP/Ad-HO-1 treatment groups as compared with controls (Figure 3). For instance, at 60 minutes of reperfusion, sGOT concentrations were $53.3 \pm 8.23 \mathrm{IU} / \mathrm{L}$ and $68.8 \pm 10.1 \mathrm{IU} / \mathrm{L}$ in the CoPP and Ad-HO-1 groups, respectively, versus $102 \pm 8.23 \mathrm{IU} / \mathrm{L}$ in untreated controls $(P<0.02)$. In 
contrast, Ad- $\beta$ Gal gene transfer did not affect the extent of $\mathrm{I} / \mathrm{R}$ insult suffered otherwise by steatotic rat livers (Figures $1-3 ; n=4$ ).

$\mathrm{ZnPP}$ abrogates the beneficial effects of HO- 1 upon hepatic $I / R$ injury. To determine if the amelioration of hepatocyte injury in this $\mathrm{I} / \mathrm{R}$ model was indeed mediated by an increase in HO-1 activity, prospective liver donors were pretreated with ZnPP, a potent HO-1 inhibitor. Unlike in the CoPP group, livers procured from obese Zucker rats pretreated with $\mathrm{ZnPP}$ alone exhibited diminished portal blood flow (Figure 1) and bile production (Figure 2), effects that were accompanied by augmented hepatocyte injury (Figure 3) comparable with otherwise untreated fatty controls. Interestingly, infusion of $\mathrm{ZnPP}$ abolished Ad-HO-1-mediated protective effects upon $\mathrm{I} / \mathrm{R}$ injury in steatotic rat livers. Therefore, portal blood flow (Figure 1) and bile production (Figure 2 ) were significantly $(P<0.05)$ decreased, and hepatocyte function became impaired (Figure 3 ) after adjunctive $\mathrm{ZnPP}$ treatment, as compared with Ad-HO-1 monotherapy.

Liver histology in the ex vivo cold ischemia model followed by reperfusion. The I/R-induced hepatocyte injury in the ex vivo model was graded using Banff's criteria (24). In the untreated fatty Zucker group, there was severe disruption of lobular architecture with marked zone 3 ballooning change, focally associated with hepatocyte necrosis (Figure 4a; Banff's score $=3.0 \pm 0.63$ ). The ZnPP-treated livers showed somewhat less lobular ballooning changes, but more sinusoidal and vascular congestion (Figure $4 \mathrm{~b}$; score $=2.86 \pm 0.12$ ). In marked contrast, CoPP-treated livers showed complete preservation of the lobular architecture with no signs of hepatocyte necrosis (Figure 4c; score $=1.21 \pm 0.39$ ). Similarly, livers transduced with Ad-HO-1 revealed focal areas of mild vacuolar degeneration with minimal hepatocyte necrosis $($ Figure $4 d$; score $=1.68 \pm 0.51)$. However, livers procured from animals treated with Ad-HO-1 plus ZnPP were characterized by severe disruption of the lobular architecture, similar to the control untreated group, with profound zone 3 ballooning change accompanied by confluent areas of hepatocyte necrosis (Figure 4e; score $=2.74 \pm 0.26$ ). Livers treated with Ad- $\beta$ Gal

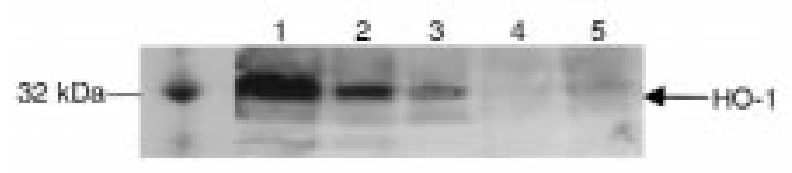

\section{Figure 5}

Western blot analysis of $\mathrm{HO}-1$ protein expression in fatty Zucker livers stored for 6 hours at $4^{\circ} \mathrm{C}$, followed by 2 hours of perfusion on the isolated perfusion rat liver apparatus. HO-1 expression was detected by using a polyclonal rabbit anti-rat $\mathrm{HO}-1 \mathrm{Ab}$. Lane 1, CoPP treatment; lane 2, Ad-HO-1 gene transfer; lane 3, Ad-HO-1 gene transfer plus $\mathrm{ZnPP}$ treatment; lane 4, ZnPP treatment; lane 5, untreated control. HO-1 migrates as a $32-\mathrm{kDa}$ protein. The relative $\mathrm{HO}-1$ expression levels analyzed by densitometer were (in absorbance units): 2.46 , $2.12,1.18,0.12$, and 0.11 for lanes $1,2,3,4$, and 5, respectively. Results shown are representative of 2-3 independent experiments.

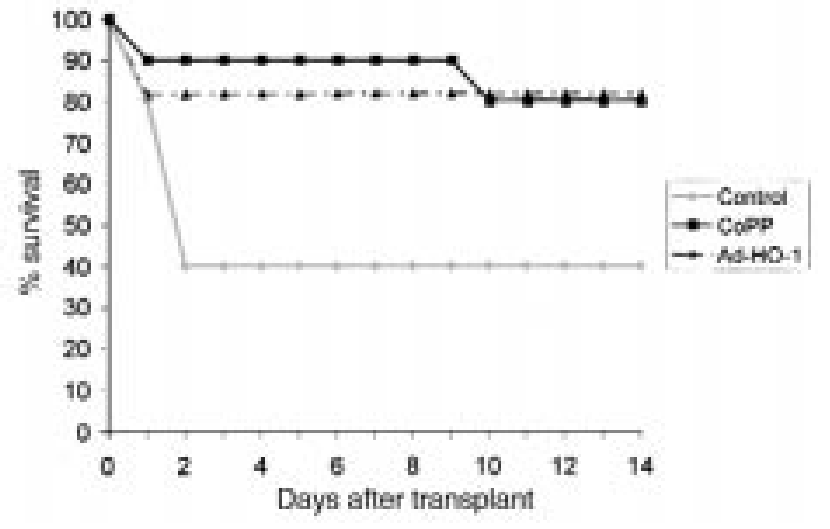

\section{Figure 6}

Prolongation of liver isograft survival. Lean Zucker rats served as recipients of liver transplants from obese Zucker donors. Donor rats were either pretreated with CoPP or Ad-HO-1 or remained untreated before liver procurement followed by 4 hours of cold ischemia. Control animal survival at 14 days was $40 \%$ versus $80 \%$ and $81.8 \%$ in the CoPP and the Ad-HO-1 group, respectively ( $n=10-11$ rats/group).

revealed less necrosis compared with the untreated group, but had severe architectural disruption and vascular congestion (Figure 4f; score $=3.0 \pm 1.41$ ).

Western analysis of HO- 1 expression in the ex vivo $I / R$ injury model. We used Western analysis to evaluate HO1 expression in liver samples following cold ischemia at the completion of 2-hour perfusion period. The relative expression levels of HO-1 protein in absorbance units (AU) were analyzed by densitometer. As shown in Figure 5, preservation of hepatic function after CoPP pretreatment or Ad-HO-1 gene transfer was accompanied by enhanced HO-1 expression (2.46 and $2.12 \mathrm{AU}$, respectively). In contrast, HO-1 was diminished after adjunctive $\mathrm{ZnPP}$ infusion (1.18 AU) and virtually undetectable in untreated (0.11 AU) and $\mathrm{ZnPP}$-pretreated $(0.12 \mathrm{AU})$ controls.

HO-1 overexpression prolongs OLT survival and improves hepatic function. We then sought to examine whether exogenous manipulation of $\mathrm{HO}-1$ expression could also confer protection against $I / R$ injury in the in vivo setting. Hence, we performed OLTs using steatotic Zucker livers that were cold stored for 4 hours before transplant into syngeneic lean Zucker rats. The treatment groups received a single dose of CoPP or Ad-HO-1 gene transfer 24 hours before liver procurement. As shown in Figure 6, recipients of liver isografts that were stored before transplantation in UW solution alone had a $40 \%$ survival rate at 14 days (4 out of 10 ). In contrast, recipients of liver isografts pretreated with CoPP showed $80 \%$ survival rate (8 out of 10). Livers pretreated with Ad-HO-1 had $81.8 \%$ survival rate at 2 weeks ( 9 out of 11 ). Indeed, 8 out of 10 lean Zucker rats engrafted with livers from CoPP-treated obese Zucker donors are still alive at well over 100 days after transplant. Prolonged survival after CoPP or Ad-HO-1 pretreatment correlated with improved OLT function as evidenced by sGOT levels. Hence, at day 1, 7, and 14 posttransplant sGOT levels 

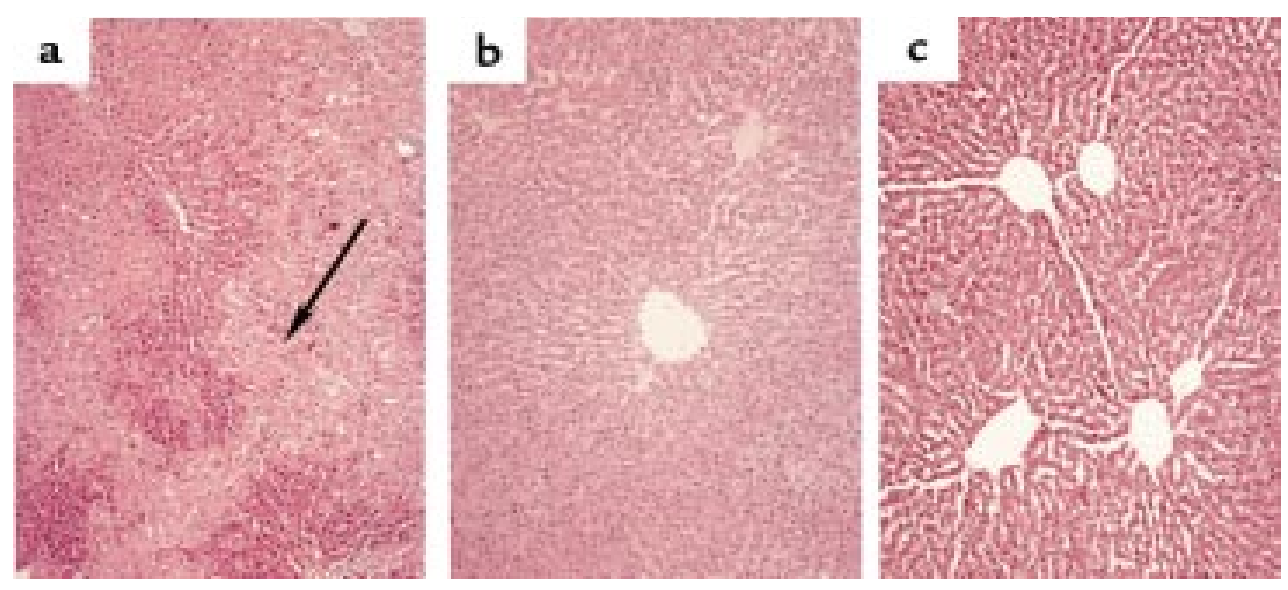

\section{Figure 7}

Photomicrographs of representative liver isografts transplanted from obese Zucker donors into lean Zucker recipients and harvested at day 1. (a) Untreated group with significant edema and pallor around periportal hepatocytes and severe disruption of lobular architecture with zone 3 necrosis (arrow; Suzuki score $=3.33 \pm 0.58$ ); (b) CoPP- and (c) Ad-HO-1-treated groups with minimal pallor, no edema, and complete preservation of lobular architecture (score $=1.33 \pm 0.70$ and $1.5 \pm 0.5$, respectively). $\times 100, \mathrm{H} \& \mathrm{E}$ stain; $n=2-3$ /group.

(IU/L) in control untreated OLTs of 2695, 1570, and 460, respectively, were significantly higher as compared with corresponding CoPP-pretreated (1838, 477, and 198, respectively; $P<0.05$ ) or Ad-HO-1-pretreated $(1628,244$, and 137, respectively; $P<0.05)$ OLTs.

Liver histology and MNC infiltration in the OLT model. Hepatocyte damage in the OLT model was assessed by a modified Suzuki's classification (25). At day 1 after transplant, control untreated liver isografts showed severe disruption of lobular architecture by ballooning change, significant edema around portal areas, and moderate to severe bile duct proliferation (Figure 7a; Suzuki score $=3.33 \pm 0.58$ ). In addition, moderate neutrophil infiltration and hepatocyte necrosis with extreme pallor that signifies glycogen depletion in the damaged hepatocytes, were prominent in this OLT group. In contrast, CoPP pretreated liver isografts at day 1 showed less neutrophil infiltration and significantly less pallor in addition to complete preservation of lobular architecture with no evidence of congestion or necrosis (Figure $7 \mathrm{~b}$; score $=1.33 \pm 0.70$ ). The Ad-HO1 -pretreated isografts showed much less neutrophil infiltration as compared with untreated controls; there was no sinusoidal congestion or hepatocyte necrosis and complete preservation of lobular architecture (Figure $7 c$; score $1.50 \pm 0.5$ ). Most histologic features characteristic for ischemic pathology resolved by $14-100$ days in those $40 \%$ of untreated OLT recipients that survived 2 weeks (not shown). However, unlike in the CoPP/Ad-HO-1-pretreated groups, untreated controls still showed significant bile duct proliferation.

Figure 8 depicts representative staining for $\mathrm{T}$ cells (a, $b, e, f)$ and macrophages $(c, d, g, h)$ in liver isografts at day $1(\mathrm{a}-\mathrm{d})$ and day $100(\mathrm{e}-\mathrm{h})$ after transplantation. Liver isografts from untreated obese Zucker donors showed massive MNC infiltration as early as at 24 hours ( $T$ cells: $9 \pm$ 3 ; monocytes/macrophages: $136 \pm 31$ ). In contrast, Zucker rats pretreated with CoPP revealed significantly decreased numbers of intragraft MNC by day 1 (T cells: $2 \pm 1$; monocytes/macrophages $71 \pm 12 ; P<0.03$ and $P<$ 0.05 , respectively). We found some heterogeneity in longterm liver grafts harvested at day 100 . Thus, about $50 \%$ of untreated grafts showed dense infiltration by $\mathrm{T}$ cells and monocytes/macrophages, followed by severe hepatocellular injury; the remainder were characterized by moderate MNC infiltration and largely preserved hepatocyte architecture. In contrast, all grafts in the CoPP group showed good preservation of hepatocyte architecture and only mild MNC infiltrate.

Western analysis of HO-1 expression in the OLT model. Finally, we employed Western blots to correlate histologic findings with local HO-1 expression in liver isografts. The relative expression levels in absorbance units were analyzed by densitometer. As shown in Figure 9, improved hepatic function after CoPP treatment was accompanied by enhanced HO- 1 expression at day $1,7,14$, and 100 after transplant (lines $1-4$, respectively; $1.21-2.14 \mathrm{AU})$. In contrast, the corresponding liver isografts from untreated Zucker rats showed little HO1 expression (lines 5-8; 0.09-0.85 AU).

\section{Discussion}

We report here the results of our studies on the protective effects of HO-1 against I/R injury in steatotic rat livers. The principal findings of this work are as follows: (a) CoPP or gene therapy-induced HO-1 overexpression prevented $\mathrm{I} / \mathrm{R}$ insult in ex vivo models of hepatic cold ischemia followed by reperfusion or syngeneic OLT; (b) HO-1 overexpression, as documented by Western blot analysis, improved liver function, preserved hepatocyte integrity, and decreased inflammatory MNC infiltration, with resultant prolongation of survival after transplantation; and (c) treatment with ZnPP, the HO-1 inhibitor, abolished these beneficial effects, documenting the direct involvement of HO-1 in protection against $\mathrm{I} / \mathrm{R}$ injury. By demonstrating, for 
the first time to our knowledge, that exogenous HO-1 induction prevents severe $\mathrm{I} / \mathrm{R}$ insult in fatty livers, our results provide the rationale for novel therapeutic approaches to maximize organ use and function through the safer use of marginal steatotic livers.

To test our hypothesis that stress-induced upregulation of HO-1 reduces I/R insult in steatotic rat livers, we have chosen 2 distinct HO-1-inducing approaches. First, donor rats were pretreated with CoPP $(5 \mathrm{mg} / \mathrm{kg}$ intraperitoneally), a regimen that

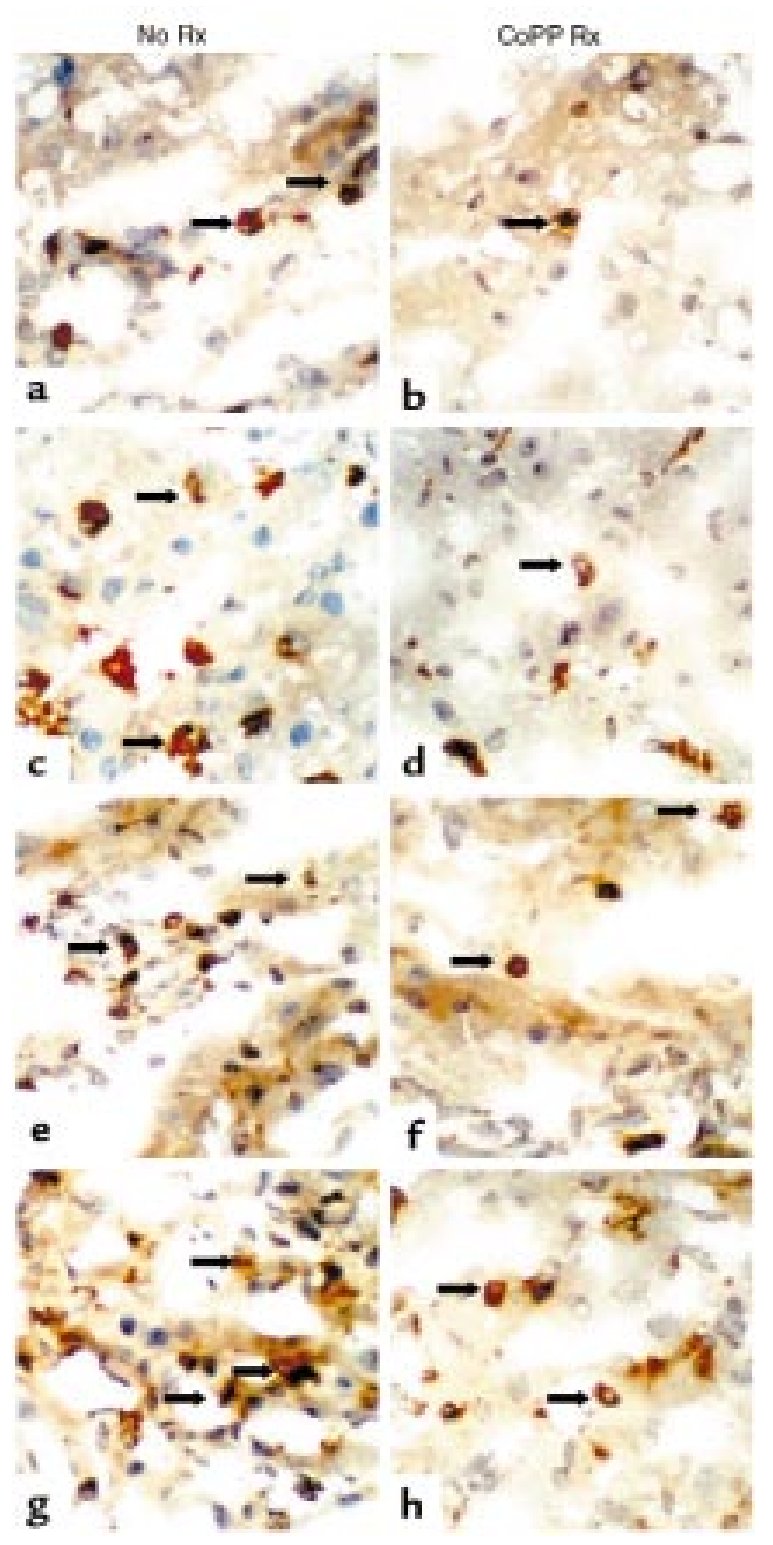

Figure 8

Immunohistochemical staining for infiltrating $T$ cells and macrophages in steatotic rat livers at day 1 and 100 following transplantation into lean Zucker recipients. Sections of liver isografts from untreated or CoPP-pretreated obese Zucker donors were stained with primary mouse $\mathrm{mAb}$ against rat $\mathrm{T}$ cells (R73) and monocytes/macrophages (ED1). (a) Control day 1 (T cells); (b) CoPP day 1 (T cells); (c) control day 1 (macrophages); (d) CoPP day 1 (macrophages); (e) control day 100 ( $T$ cells); (f) CoPP day 100 (T cells); (g) control day 100 (macrophages); (h) CoPP day 100 (macrophages). Original $\times 272$. increases HO- 1 protein levels in rat livers by $250 \%$ in a rat sandwich ELISA (R. Buelow, unpublished data). In our present study, a single dose of CoPP induced a prolonged increase in HO-1 expression that lasted more than 100 days after OLT. Second, because infusion of CoPP in high doses may modulate other heme enzymes such as nitric oxide synthase (NOS) and guanylate cyclase $(28,29)$, we have also used Ad-based gene delivery to provide "proof of principle" and to selectively upregulate $\mathrm{HO}-1$ expression in prospective liver donors. Indeed, as recently shown in a hyperoxia-induced lung injury rat model (30), our Western blot analysis confirmed increased $\mathrm{HO}-1$ protein expression in the ex vivo I/R model using Ad-HO1 -transduced rat steatotic livers.

Ischemia/reperfusion is markedly increased in steatotic livers compared with normal livers, and reactive oxygen species have been shown to contribute, at least in part, to this event (31). Moreover, the antioxidant defenses of hepatocytes in steatotic livers are decreased in comparison with normal livers (32). Exogenous upregulation of HO-1 in our study prevented or significantly decreased hepatic injury in 2 clinically relevant and well-defined ex vivo rat fatty liver models of cold ischemia followed by reperfusion or syngeneic OLT. The beneficial effects in the ex-vivo I/R-injury model were reflected by the ability of exogenously upregulated HO1 to improve portal vein blood flow, increase bile production, and depress sGOT levels, all well-accepted parameters of hepatic function (6). Portal blood flow is mostly affected by resistance in the graft caused by lobular ballooning, hepatocyte swelling, and sinusoidal congestion. In this ex vivo perfusion model, the improved portal venous blood flow represents less hepatocyte injury and lobular disarray in the liver rather than the endothelium-dependant vasodilatory effects of carbon monoxide. In the in vivo liver isotransplant model, enhanced HO-1 expression improved animal survival from $40 \%$ in untreated controls to about $80 \%$ after CoPP treatment or local Ad-HO-1 gene delivery, an ultimate test for the liver function. Collectively, these results are consistent with the ability of $\mathrm{HO}-1$ to protect cells from oxidative injury (33-35).

Upregulation of HO-1 inhibits inflammatory responses (36-38) consistent with our present immunohistochemical findings of markedly decreased MNC infiltration in CoPP-pretreated liver isografts, as compared with untreated controls. Whereas it is still unclear how HO-1 upregulation influenced graft infiltration, several possibilities can be envisioned: (a) decreased production of cytokines and chemokines by infiltrating cells may have prevented local cell sequestration at the graft site; (b) reduced cytokine levels could diminish endothelial cell activation; and (c) HO-1 may have influenced directly endothelial cell activation. Indeed, in our ongoing real-time RT-PCR studies, markedly diminished expression of mRNA coding for Th1-type IFN- $\gamma / \mathrm{IL}-2$ as well as Th2-type IL-10 was readily detectable in HO-1overexpressed and functioning liver isografts despite 


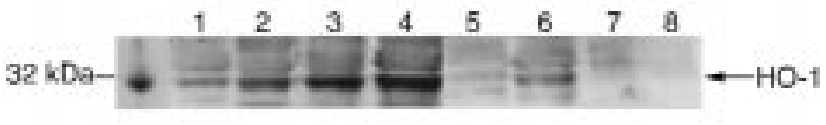

Figure 9

Western blot analysis of $\mathrm{HO}-1$ protein expression in fatty livers with or without CoPP pretreatment. Livers were stored for 4 hours at $4^{\circ} \mathrm{C}$, transplanted into lean Zucker recipients, and then harvested at 1, 7, 14 , and 100 days. The expression of HO- 1 was detected by a polyclonal rabbit anti-rat HO-1 Ab. Lane 1, CoPP day 1 ; lane 2, CoPP day 7; lane 3, CoPP day 14; lane 4, CoPP day 100; lane 5, untreated day 1 ; lane 6 , untreated day 7 ; lane 7 , untreated day 14 ; lane 8 , untreated day 100. HO-1 migrates as a $32-\mathrm{kDa}$ protein. The relative $\mathrm{HO}-1$ expression levels analyzed by densitometer were (in absorbance units): 1.21, 1.27, 1.63, and 2.14 for CoPP-treated livers in lanes 1, 2, 3, and 4, respectively. The HO-1 expression for untreated controls in lanes 5, 6, 7, and 8 were (in absorbance units): $0.09,0.17,0.85$, and 0.75 , respectively. Data shown are representative of 3 independent experiments.

previous I/R injury (F. Amersi, unpublished data). Moreover, consistent with our preliminary findings in the I/R steatotic OLT model, we have recently shown that CoPP treatment suppressed both proliferative responses and elaboration of TNF- $\alpha$, IFN- $\gamma$, and IL-10 in a murine cardiac allograft model (14). CoPP-mediated in vivo effects, such as HO-1 upregulation, inhibition of T-cell proliferation, cytokine elaboration, T- and NK-cell cytotoxicity, and prolongation of allograft survival (14) were all observed after treatment with an immunomodulatory MHC Class I-derived peptide (D2702.75-84) (33). Most recently, we have also shown that a rationally designed HO-1-inducing immunomodulatory peptide (RDP 1258) inhibited rat renal transplant vasculopathy (36). Moreover, in agreement with the current report, our ongoing studies show that CoPP-triggered HO-1 overexpression in small-bowel donors may decrease preservation/reperfusion injury and improve survival of both the animal and of the transplanted bowel (39). Clearly, our present findings support the idea that HO-1 upregulation does not associate just with exogenous immunosuppression, but it may well represent an essential component of stress-mediated immunomodulation. To prove this hypothesis, we are currently generating HO-1 transgenic rats to use as liver donors and/or recipients in our hepatic I/R injury models.

The results of our experiments in which ZnPP-mediated inhibition of HO-1 negated beneficial effects seen in vivo after CoPP treatment or Ad-HO-1 gene transfer endorse the hypothesis that the mechanism behind protection against hepatic I/R injury involves $\mathrm{HO}-1$ induction rather than modulation of other biochemical pathways that may protect hepatocytes from oxidative injury. In other studies rat epithelial cells transfected with HO-1 cDNA exhibited marked increases of HO-1 protein and resistance against hyperoxia, whereas inhibition of HO-1 with tin protoporphyrin (SnPP) ablated protection against hyperoxia (40). Similarly, in rodent models of renal failure, elevated $\mathrm{HO}-1$ expression reduced tissue injury $(41,42)$. Again, protection could be reversed by SnPP-induced inhibition of HO-1. In ani- mal models of inflammatory disease, enhanced HO-1 activity downregulated inflammation, whereas inhibition of $\mathrm{HO}$ exacerbated the inflammatory response (37, 38). In septic shock models, increased HO-1 activity protected animals from LPS-induced death (43). Consistent with these observations, HO-1-deficient mice suffer from progressive chronic inflammatory disease and are extremely sensitive to stressful injury (44). Similar observations were made with the first known human case of HO-1 deficiency (45).

The mechanisms behind HO-1-mediated cytoprotection remain unclear. However, all of the end products of heme degradation, including biliverdin, bilirubin, and $\mathrm{CO}$, are known to modulate immune effector functions (46-48). Biliverdin has also been shown to inhibit hu-man complement in vitro (46). Bilirubin inhibits human lymphocyte responses, including PHAinduced proliferation, IL-2 production, and antibodydependent and -independent cell-mediated cytotoxicity $(44,45)$. Moreover, because of the heme protein nature of NOS, induction of HO-1 is likely to modulate nitric oxide (NO) production, an important effector molecule involved in inflammation and immune regulation (49). Indeed, HO-1 upregulation correlates with increased production of $\mathrm{NO}$, which in turn may inhibit lymphocyte proliferation following CoPP therapy, as in our present studies. On the other hand, NO is also known to induce HO-1 expression. This effect may be of significance because $\mathrm{CO}$ directly inhibits NOS activity by binding to the heme moiety of the NOS enzyme and thus downregulating NO production. Like NO, CO contributes to endotheliumdependent vasodilatation and inhibits platelet aggregation by elevating intracellular cGMP levels (50). The deleterious effects of hyperoxia are thought to be mediated by reactive oxygen species (ROS). Both biliverdin and bilirubin are efficient peroxyl radical scavengers that inhibit lipid peroxidation (51). Bilirubin scavenges peroxyl radicals as efficiently as $\alpha$-tocopherol, which is regarded as the most potent antioxidant of lipid peroxidation. On the other hand, oxygen radicals may trigger cascade of antiapoptotic events, including those that involve activation of bcl- 2 protooncogene. Indeed, as shown by us (15) and others $(13,16)$, increased expression of bcl-2 may represent one of the mechanisms by which increased HO-1 expression may promote protection against tissue injury. All these factors point to a complex picture of putative regulatory interactions between the $\mathrm{HO}$ system and the host cytokine network set in motion through the biological activity of heme degradation products.

In conclusion, CoPP- or gene therapy-induced HO1 overexpression protects against severe I/R injury in steatotic rat liver models of ex vivo cold ischemia followed by reperfusion or OLT. To our knowledge, this is the first report that documents the potential utility of HO-1 in increasing the donor transplant pool through modulation of marginal steatotic livers or conditions of prolonged ischemia. Our findings raise 
the possibility of refined new treatment regimens in OLT that may ultimately improve the overall success of liver transplantation.

\section{Acknowledgments}

The authors thank Lou Ignarro for helpful discussion and critical reviewing of the manuscript. This work was supported by National Institutes of Health grants RO1 AI-23847, RO1 AI-42223, Dumont Research Foundation, and Sangstat Medical Corp.

1. Markin, R.S., et al. 1993. Frozen section evaluation of donor livers before transplantation. Transplantation. 56:1403-1409.

2. Hornboll, P., and Olsen, T.S. 1982. Fatty changes in the liver: the relation to age, overweight, and diabetes mellitus. Acta Path. Microbiol. Immunol. Scand. [A.] 90:199-205.

3. D’Allessandro, A.M., Kalayoglu, M., and Sollinger, H.W. 1991. The predictive value of donor liver biopsies for the development of primary nonfunction after orthotopic liver transplantation. Transplantation. 51:157-162.

4. Strasberg, S.M., Howard, J.K., Melmenti, E.P., and Hersh, M. 1994. Selecting the donor liver: risk factors for poor function after orthotopic liver transplantation. Hepatology. 20:829-838.

5. Fellstrom, B., Akuyrek, L.M., and Zezina, L. 1998. Post ischemic reperfusion injury and allograft arteriosclerosis. Transplant. Proc. 38:4278-4280.

6. Dulkanchainun, T.S., et al. 1998. Reduction of hepatic ischemia/reperfusion injury by a soluble p-selectin glycoprotein ligand-1. Ann. Surg. 227:832-840.

7. Thurman, R.G., et al. 1988. Hepatic reperfusion injury following orthotopic liver transplantation in the rat. Transplantation. 46:502-506.

8. Serizawa, A., Nakamure, S., Suzuki, S., Baba, S., and Nakano, M. 1996 Involvement of platelet activating factor in cytokine production and neutrophil activation after hepatic ischemia reperfusion. Hepatology. 23:1656-1663.

9. Goode, H.F., et al. 1994. Reperfusion injury, antioxidants and hemodynamics during OLT. Hepatology. 19:354-360

10. Shau, H., and Kim, A. 1994. Identification of natural killer enhancing factor as a major antioxidant in human red blood cells. Biochem. Biophys. Res. Commun. 199:83-87.

11. Maines, M.D. 1992. Heme oxygenase: clinical applications and functions. CRC Press. Boca Raton, FL. 276 pp.

12. Rodgers, P.A., Seidman, P.S., Wei, P.L., Denney, P.A., and Stevenson, D.C. 1996. Duration of action and tissue distribution of zinc protoporphyrin in neonatal rats. Pediatr. Res. 39:1041-1049.

13. Soares, M.P., et al. 1998. Expression of HO-1 can determine cardiac xenograft survival. Nat. Med. 4:1073-1077.

14. Woo, J., et al. 1998. Stress protein-induced immunosuppression: inhibition of cellular immune effector functions following overexpression of haem oxygenase (HSP 32). Transpl. Immunol. 6:84-93.

15. Hancock, W.W., Buelow, R., Sayegh, M.H., and Turka, L.A. 1998. Antibody induced transplant arteriosclerosis is prevented by graft expression of anti-oxidant and anti-apoptotic genes. Nat. Med. 12:1392-1396.

16. Panahian, N., Yoshiura, M., and Maines, M.D. 1999. Overexpression of heme oxygenase- 1 is neuroprotective in a model of permanent middle cerebral artery occlusion in transgenic mice. J. Neurochem. 72:1187-1203.

17. Shibahara, S., Muller, R., Taguchi, H., and Yoshida, T. 1985. Cloning and expression of cDNA for rat heme oxygenase. Proc. Natl. Acad. Sci. USA. 93:10393-10398

18. Graham, F.L., and Prevec, L. 1992. Manipulation of adenoviral vectors in molecular biology. Gene transfer and expression protocols. Biotechnology. 20:363-390.

19. Gomez-Foix, A.M., et al. 1992. Adenovirus mediated gene transfer of the muscle glycogen phosphorylase gene into hepatocyte confers altered regulation of glycogen metabolism. J. Biol. Chem. 267:25129-25134.

20. Fallaux, F.J., et al. 1996. Characterization of 911: a new helper cell line for the titration and propagation of early region I-deleted adenoviral vectors. Hum. Gene Ther. 7:215-222.

21. McGrory, W.J., Bautista, D.S., and Graham, F.L. 1988. A simple technique for the rescue of early region 1 mutations into infectious human adenovirus type 5. Virology. 163:614-617.

22. Kolls, J., Peppel, K., Silva, M., and Beutler, B. 1994. Prolonged and effective blockade of tumor necrosis factor activity through adenovirus-mediated gene transfer. Proc. Natl. Acad. Sci. USA. 91:215-219.

23. Kamada, N., and Calne, R.Y. 1979. Orthotopic liver transplantation in the rat: technique using cuff for portal vein anastomosis and biliary drainage. Transplantation. 28:47-48.

24. Demetris, A.J., et al. International Banff Schema Consensus Conference. The Third Banff Conference on Allograft Pathology. June 21-25, 1995.
Banff, Alberta, Canada.

25. Suzuki, S., Toledo-Pereyra, L.H., Rodriguez, F.J., and Cejalvo, D. 1993. Neutrophil infiltration as an important factor in liver ischemia and reperfusion injury. Transplantation. 55:1265-1272.

26. Coito, A.J., Binder, J., de Sousa, M., and Kupiec-Weglinski, J.W. 1994. The expression of extracellular matrix proteins during accelerated rejection of cardiac allografts in sensitized rats. Transplantation. 57:599-605.

27. Coito, A.J., et al. 1995. Anti-TNF- $\alpha$ antibody treatment downregulates the expression of fibronectin and decreases cellular infiltration of cardiac allografts in rats. J. Immunol. 154:2949-2958.

28. Ignarro, L.J., Ballot, B., and Woods, K.S. 1984. Regulation of soluble guanylate cyclase activity by porphyrins and metalloporphyrins. J. Biol. Chem. 259:6201-6207.

29. Wolff, D., Naddelman, R.A., Lubeskie, A., and Sulcs, D.A. 1996. Inhibition of nitric oxide synthase isoforms by porphyrins. Arch. Biochem. Biophys. 333:27-34.

30. Otterbein, L.E., et al. 1999. Exogenous administration of heme oxygenase- 1 by gene transfer provides protection against hyperoxia induced lung injury. J. Clin. Invest. 103:1047-1054.

31. Taneja, C., Prescott, L., and Koneru, B. 1998. Critical preservation injury in rat fatty livers is to hepatocytes, not sinusoidal lining cells. Transplantation. 65:167-172

32. Gonzales-Flecha, B., Cutrin, J.C., and Bovens, A. 1993. Time course and mechanism of oxidative stress and tissue damage in rat liver subjected to ischemia reperfusion. J. Clin. Invest. 91:456-460.

33. Iyer, J., et al. 1998. Characterization and biological significance of immunosuppressive peptide D2702.75-84 (E-V) binding protein. J. Biol. Chem. 273:2692-2697.

34. Nath, K.A., et al. 1994. The functional significance of the induction of heme oxygenase by oxidative stress. J. Lab. Clin. Med. 123:461-463.

35. Nath, K.A., et al. 1992. Induction of heme oxygenase is a rapid, protective response in rhabdomyolysis in the rat. J. Clin. Invest. 90:267-270.

36. Parry, N., Buelow, R., Jiang, J., Garcia, B., and Zhong, R. 1999. A rationally designed immunomodulatory peptide upregulates expression of hemoxygenase- 1 and attenuates chronic rejection in a rat renal allograft model. Transplantation. 67:S252.

37. Laniado-Schwartzman, M., et al. 1996. Heme oxygenase induction with attenuation of experimentally induced corneal inflammation. Biochem. Pharmacol. 53:1069-1073.

38. Willis, D., Moore, A.R., Frederick, R., and Willoughby, D.A. 1996. Heme oxygenase: a novel target for the modulation of the inflammatory response. Nat. Med. 2:87-90.

39. Squiers, E.C., Bruch, D., Buelow, R., and Tice, D.G. 1999. Pretreatment of small bowel isograft donors with cobalt-protoporphyrin decreases preservation injury. Transplant. Proc. 31:585-589.

40. Lee, P.J., Alam, J., Wiegand, G.W., and Choi, A.M.K. 1996. Overexpression of hemoxygenase- 1 in human pulmonary epithelial cells results in cell growth arrest and increased resistance to hyperoxia. Proc. Natl. Acad. Sci. USA. 93:10393-10399.

41. Vogt, B.A., et al. 1996. Glomerular inflammation induces resistance to tubular injury in the rat. J. Clin. Invest. 98:2139-2145.

42. Agarwal, A., Balla, J., Alam, J., Croatt, J., and Nath, K.A. 1995. Induction of heme oxygenase $(\mathrm{HO})$ in renal injury: a protective role in acute cisplatin nephrotoxicity in the rat. Kidney Int. 48:1298-1307.

43. Otterbein, L., Sylvester, S.L., and Choi, A.M.K. 1995. Hemoglobin provides protection against lethal endotoxemia in rats: the role of heme exygenase-1. Am. J. Respir. Cell Mol. Biol. 13:595-602.

44. Poss, K.D., and Tonegawa, S. 1997. Reduced stress defense in heme oxygenase 1 deficient cells. Proc. Natl. Acad. Sci. USA. 9:10925-10930.

45. Yachie, A., et al. 1999. Oxidative stress causes enhanced endothelial cell injury in human heme oxygenase-1 deficiency. J. Clin. Invest. 103:129-135.

46. Nagakami, T., Toyomura, K., Kinoshita, T., and Morisawa, S. 1993. A beneficial role of bile pigments as an endogenous tissue protector: Anticomplement effects of biliverdin and conjugated bilirubin. Biochem. Biophys. Acta. 1158:189-193.

47. Haga, Y., Temepero, A.M., Kay, D., and Zetterman, R.K. 1996. Intracellular accumulation of unconjugated bilirubin inhibits phytohemagglutinin-induced proliferation and interleukin-2 production of human lymphocytes. Dig. Dis. Sci. 41:1468-1474.

48. Haga, Y., Tempero, M.A., and Zetterman, R.K. 1996. Unconjugated bilirubin inhibits in vitro major histocompatibility complex-unrestricted cytotoxicity in human lymphocytes. Biochem. Biophys. Acta. 1316:29-34

49. Maines, M.D. 1997. The heme oxygenase system: a regulator of second messenger gases. Annu. Rev. Pharmacol. Toxicol. 37:517-554.

50. Brune, B., and Ulrich, V. 1987. Inhibition of platelet aggregation by carbon monoxide is mediated by activation of guanylate cyclase. Mol. Pharmacol. 32:497-504.

51. Neuzil, J., and Stocker, R. 1994. Free and albumin-bound bilirubin are efficient antioxidants for $\alpha$-tocopherol, inhibiting plasma and low-density lipoprotein peroxidation. J. Biol. Chem. 269:16712-16719. 